\section{Jahre Universitäts-Hautklinik Jena}

\author{
Zur Dynamik der wissenschaftlichen Leistungsent- \\ wicklung am Beispiel der Wissenschaftspublizistik
}

Zusammenfassung. Wissenschaftliche Publizistik ist ein Maß der wissenschaftlichen Kompetenz und Effizienz einer Universitäts-Einrichtung. Anhand der Analyse von Publikationsdaten soll die jüngere Wissenschaftsgeschichte der Klinik für Dermatologie und Allergologie der Jenaer Universität aus Anlass des 75. Jahres ihres Bestehens dargestellt werden. Es kann auf diese Weise belegt werden, dass nicht allein der Umfang wissenschaftlicher Aktivitäten, sondern vor allem die Qualität eine bemerkenswerte Steigerung erfahren hat. Wichtige Qualitätssprünge fallen mit der Wiedervereinigung Deutschlands zusammen. In ihrem 75. Jahr präsentiert sich die Klinik als eine der produktivsten im Osten Deutschlands.

75-Year Anniversary of the Department of Dermatology, Jena - On the Dynamics of Scientific Publication. The publication of scientific work is a tool to measure the scientific competence and efficacy of university departments. Using an analysis of scientific publications of the years 1970 to 1999 the recent history of science at the Department of Dermatology and Allergology of the University of Jena has been illustrated in honour of the 75 anniversary of its foundation. One can show that not only the ouput of scientific work has been increased but also quality. Of major impact was the re-unification of Germany. Nowadays, the Department is one of the most productive in Eastern Germany.

1922 wurde der Grundstein zur Klinik für Haut- und Geschlechtskrankheiten der damaligen Thüringer Landesuniversität in der Erfurter Straße gelegt. 1924 nahm die Klinik ihre Arbeit auf. Dieses Gebäude beherbergt nunmehr 75 Jahre die Universitäts-Hautklinik Jena [4]. Seit 1999 trägt die Klinik laut Strukturplan der Fakultät die Bezeichnung „Klinik für Dermatologie und Allergologie“. Während in vorangegangenen Publikationen ein ausführlicherer geschichtlicher Abriss zur Entwicklung der Dermatologie, zu den Ordinarien und dem Aufbau der Klinik gegeben wurde [12,13], soll in der vorliegenden Arbeit das Augenmerk auf die jüngste Geschichte gelegt werden. Insbesondere gilt es, die wissenschaftliche Publizistik zu analysieren, die ein wichtiges, wenn auch nicht unumstritte-

Akt Dermatol 2001; 27: 215-218

(c) Georg Thieme Verlag Stuttgart · New York ISSN 0340-2541

\section{U. Wollina, P. Elsner}

Klinik für Dermatologie und Allergologie der FriedrichSchiller-Universität Jena (Direktor: Prof. Dr. P. Elsner)

nes Maß der wissenschaftlichen Produktivität verkörpert $[2,5,6]$. Wissenschaftliche Publizistik macht Forschung öffentlich. Moderne Datenbanken ermöglichen Vergleiche und Analysen.

\section{Methodik}

Als bibliografische Grundlage dienten die internationale Datenbank MEDLINE sowie die jährlichen Forschungsberichte der Klinik 1970-99. Bücher und Monografien, Habilitationsund Dissertationsschriften fanden keine Berücksichtigung. Die Qualitätsbeurteilung erfolgt mit dem Impact-Faktor (ISI Philadelphia). Der Einfachheit halber wird für die Berechnungen der Impact-Faktor von 1997 zugrunde gelegt. Für die Vergleiche unterschiedlicher Kliniken werden ausschließlich die in MEDLINE aufgeführten Arbeiten verwendet, obwohl sich daraus eine Unterbewertung ergeben kann. So findet sich beispielsweise die Zeitschrift "Allergologie“ nicht in MEDLINE, obwohl sie einen Impact-Faktor besitzt und von den allergologischen Arbeitsgruppen Deutschlands häufig für Publikationen genutzt wird. Statistische Auswertungen erfolgen mittels zweiseitigem t-Test. Ein $\mathrm{p}<0,05$ wird als signifikant angesehen. Korrelationen wurden mit der Methode nach Pearson errechnet.

\section{Ergebnisse \\ Publikationszahl}

In den Jahren 1970 - 1990 lag die durchschnittliche jährliche Publikationszahl bei 13 in MEDLINE erfassten Arbeiten ( Maximum 29 Arbeiten 1990). 1992 bis 1996 wurden insgesamt 25 Arbeiten in MEDLINE erfasst (Durchschnitt 5). Dieser Abfall ist vor allem durch das Auslaufen der regelmäßigen Publikation der Dermatologischen Monatsschrift als eigenständiger Zeitschrift zu erklären. Einen deutlichen Zuwachs gab es in den Jahren 1997-1999 mit insgesamt 119 Arbeiten (19971999 i. Vgl. zum Zeitraum 1970 - 1996; p <0,001).

Das Verhältnis von MEDLINE-referierten Artikeln zur Gesamtzahl wissenschaftlicher Publikationen lag 1999 bei 1:2, 1989 bei 1:10. Der Anteil der Beiträge in Journalen mit einem Impact-Faktor $>1$ stieg rascher an als die Zahl aller MEDLINE-referierten Artikel. 


\section{Wissenschaftliche Schwerpunkte}

In den Jahren 1970 bis 1990 wurden drei Viertel aller Arbeiten auf klinischem Gebiet verfasst, ein Viertel widmete sich eher experimentellen Themen. Die Forschung auf dem Gebiet der Kollagenosen und der Psoriasis hatte in den frühen 70er Jahren die tragende Rolle gespielt. Mit dem Weggang der Arbeitsgruppe von N. Sönnichsen nach Berlin brach die Entwicklung ab. Erst Mitte der 80er Jahre waren diese Arbeitsgebiete wieder zu den wichtigsten Forschungsschwerpunkten der Klinik geworden (Knopf). Weiterhin bestand in den späten 70er und frühen 80er Jahren eine kleine onkologische Arbeitsgruppe (Wätzig, Knopf).

Den deutlichsten Zuwachs an Publikationszahlen zeigte die Andrologie/Sexualmedizin (1970-1975 - <1\%; 1976- 1980 $8 \%$ ) unter E. Günther. Auch in den letzten Jahren hatte die Arbeitsgruppe trotz personeller Verkleinerung einen stabilen Anteil an der wissenschaftlichen Aktivität der Klinik (Schreiber).

Mit der Wiedervereinigung Deutschlands setzte eine komplette Veränderung der Rahmenbedingungen wissenschaftlichen Arbeitens ein. Geht man lediglich nach der Anzahl der erfassten Publikationen, so zeigten die Gebiete Allergologie/Berufsdermatologie (Elsner) und Onkologie/operative Dermatologie (Wollina) den deutlichsten Zuwachs. Im Zeitraum von 1997 1999 wird die allergologisch/berufsdermatologisch ausgerichtete Forschung zur tragenden Säule der Klinik. Allein im letzten Jahr finden sich zu diesen Themen 20 Publikationen, größtenteils in MEDLINE referiert (Onkologie/Wundheilung - 15; Andrologie/Endokrinologie - 4).

Es haben sich die folgenden Felder als Kernkompetenzen erwiesen: Allergologie/Berufsdermatologie/Hautphysiologie, Dermatologische Onkologie/Wundbehandlung und Andrologie/Endokrinologie. Ausgehend von den Kernkompetenzen sind neue Arbeitsgebiete etabliert worden, die teils eine erhebliche Dynamik kennzeichnet: Telemedizin, Vulva- und Haarerkrankungen, Dermatopharmakologie und Dermatokosmetik. Dem Gebiet Haare/Nägel waren 1997-1999 allein 8 Arbeiten gewidmet.

\section{Qualität der Publikationen}

Ein direkter Vergleich ist über den Zeitraum von 1970-99 schwierig. Zum einen waren die Publikationsmöglichkeiten in der ehemaligen DDR durch Restriktionen erheblich eingeschränkt und die Auswahl des Publikationsorgans nach Wichtung des Impact-Faktors spielte seinerzeit keine Rolle. Zum anderen hatten sich die materiellen Grundlagen der Forschung an den Kliniken besonders in den 80er Jahren zunehmend verschlechtert.

Von 1970 - 1990 erschien die ganz überwiegende Zahl der Arbeiten in der Dermatologischen Monatsschrift und in deutschsprachigen Zeitschriften. In den letzten drei Jahren wurden bereits über $50 \%$ aller Arbeiten in internationalen Journalen publiziert. Die Liste der am häufigsten genutzten Journale zeigt Tab. 1.

Für den Impact-Faktor ergibt sich folgendes Bild: In den Jahren von 1970 bis 1975 lag der durchschnittliche jährliche kumulative Impact-Faktor (JKIF) unter 5, er fiel in der zweiten Hälfte
Tab. 1 Die Journale, in denen die Mitarbeiter der Universitäts-Hautklinik Jena 1997-1999 am häufigsten publizierten. Bei gleicher Publikationszahl erhält die Zeitschrift mit dem höheren Impact-Faktor den höheren Rang. Impact-Faktor 1997

\begin{tabular}{|c|c|c|c|}
\hline Rang & Journal & $\begin{array}{l}\text { Impact- } \\
\text { Faktor }\end{array}$ & $\begin{array}{l}\text { Zahl der } \\
\text { Publikationen }\end{array}$ \\
\hline 4 & Acta Dermato-Venereologica & 1,091 & 7 \\
\hline 7 & Allergy & 2,015 & 5 \\
\hline 9 & British Journal of Dermatology & 1,838 & 5 \\
\hline 2 & Contact Dermatitis & 1,130 & 12 \\
\hline 1 & Dermatology & 0,719 & 16 \\
\hline 3 & Der Hautarzt & 0,487 & 9 \\
\hline 5 & $\begin{array}{l}\text { Deutsche Medizinische } \\
\text { Wochenschrift }\end{array}$ & 0,756 & 7 \\
\hline 10 & $\begin{array}{l}\text { Journal of Investigative } \\
\text { Dermatology }\end{array}$ & 4,584 & 4 \\
\hline 6 & Pediatric Dermatology & 0,381 & 6 \\
\hline 8 & $\begin{array}{l}\text { Journal of the American } \\
\text { Academy of Dermatology }\end{array}$ & 1,891 & 5 \\
\hline
\end{tabular}

der 70er Jahre auf nahe Null (JKIF < 1). Erst Mitte der 80er Jahre erschienen wieder einzelne Beiträge in internationalen Journalen (JKIF 1983 - $1991<5$ ).

1991 - 1994 hatte sich ein nahezu kompletter Personalwechsel vollzogen, der zunächst das Interesse auf den Erhalt der klinischen Leistungsfähigkeit lenkte und das Interesse an wissenschaftlicher Arbeit minderte. Dennoch kam es 1992 zu einem gewissen Aufschwung und der kumulative JKIF stieg über 8. In der zweiten Hälfte der 90er ergibt sich ein deutlich positiveres Bild mit einem durchschnittlichen JKIF (1997-1999) von 49 (Differenz zu 1970-1991 hochsignifikant; zweiseitiger tTest; $\mathrm{p}<0,001)$.

\section{Wo wird publiziert?}

Dermatologische Arbeiten wurden ganz überwiegend in dermatologischen Zeitschriften publiziert. Vor 1991 sind etwa 80\% der MEDLINE-referierten Arbeiten in der Dermatologischen Monatsschrift erschienen. Auch in den 90ern dominieren dermatologische Zeitschriften (Tab.1), was die Anzahl der Publikationen betrifft. Die Vielfalt hat allerdings zugenommen. Ein deutlicher Anteil von 38\% des JKIF rekrutiert sich jedoch aus Publikationen in nicht-dermatologischen Journalen. Die drei am häufigsten genutzten Zeitschriften der Jahre 1970 - 1999 sind Tab. 2 zu entnehmen.

Die folgenden Zeitschriften trugen mehr als 9 Punkte zum kumulativen Impact-Faktor der Jahre 1997 - 1999 bei: Journal of Investigative Dermatology (18,336), Acta Dermato-Venereologica (7,637), Journal of Clinical Investigation (9,667), Journal of the American Academy of Dermatology $(9,455)$.

\section{Diskussion}

Das wissenschaftliche Renommee dermatologischer Kliniken ist für das Selbstverständnis des Fachgebietes von ebenso großer Bedeutung wie für die Position innerhalb der medizinischen Disziplinen $[1,2,4-6,10]$. 
Tab. 2 Die am häufigsten genutzten MEDLINE-referierten Publikationsorgane für die Universitäts-Hautklinik Jena in den Jahren 19701999

\begin{tabular}{ll}
\hline Jahrgänge & Zeitschriften \\
\hline $1970-1975$ & $\begin{array}{l}\text { Dermatologische Monatsschrift, Das Deutsche } \\
\text { Gesundheitswesen, Acta Biologica Medica }\end{array}$ \\
$1976-1980$ & $\begin{array}{l}\text { Dermatologische Monatsschrift, Das Deutsche } \\
\text { Gesundheitswesen, Zentralblatt Gynäkologie }\end{array}$ \\
$1980-1985$ & $\begin{array}{l}\text { Dermatologische Monatsschrift, Hautarzt, } \\
\text { Allergie und Immunologie (Leipzig) }\end{array}$ \\
$1986-1990$ & $\begin{array}{l}\text { Dermatologische Monatsschrift, Zeitschrift für } \\
\text { Hautkrankheiten, Archives of Dermatological } \\
\text { Research }\end{array}$ \\
$1991-1995$ & $\begin{array}{l}\text { Hautarzt, Archives of Dermatological Research, } \\
\text { Histology and Histopathology }\end{array}$ \\
$1996-1999$ & Dermatologica, Contact Dermatitis, Hautarzt \\
\hline
\end{tabular}

Die Nutzung internationaler Datenbasen erlaubt es, die Dynamik der Veränderungen des Publikationsverhaltens und der Qualität der Veröffentlichungen zu analysieren [1-4]. Dabei ist weniger die Zahl der Publikationen ausschlaggebend als vielmehr bibliometrische Werkzeuge wie der Impact-Faktor, der Science Impact Index und der Zitations-Index [2,4,8]. Eine fachspezifische Normung ist sinnvoll. So lag der mittlere Science Impact Index für westdeutsche Dermatologen 1990 bei $2,5[8]$.

Über Sinn und Unsinn dieser Werte ist an anderer Stelle ausführlich diskutiert worden $[2,3,7,8]$. Die Verwendung des Impact-Faktors zum Vergleich von Wissenschaftlern und Institutionen ist nicht ohne Probleme und Widersprüche. „Impact factor is not a perfect tool to measure the quality of articles but there is nothing better and it has the advantage of already being in existence and is, therefore, a good technique for scientific evaluation“ [4].

Von speziellem Interesse ist eine Publikationsanalyse, wenn sich innerhalb kurzer Zeiträume die Bedingungen, unter denen Wissenschaft ausgeübt wird, ändern. Dies war für das Gebiet der ehemaligen DDR durch den Fall der Mauer und die Wiedervereinigung Deutschlands der Fall. Exemplarisch sollte an der jüngeren Wissenschaftsgeschichte der Universitäts-Hautklinik Jena dieser Wandel dargestellt werden. Hinsichtlich ihrer Größe, des Personalbestandes und der technischen Ausstattung war sie mit anderen Universitäts- und Akademie-Kliniken der ehemaligen DDR vergleichbar. Sie zählte allerdings zu den kleineren Häusern bezüglich ihrer Bettenzahl.

Bei guter Ausgangsposition Anfang der 70er Jahre hatte die wissenschaftliche Publizistik in der zweiten Hälfte des Jahrzehnts einen Tiefpunkt erreicht. In den 80er Jahren erhöhte sich zwar die Zahl der Publikationen [11], zu einer signifikanten Steigerung der wissenschaftlichen Qualität gemessen am kumulativen Impact-Faktor kam es jedoch erst in den 90ern. Mit der Konzentration auf Kernkompetenzen der Klinik, materielle Verbesserungen in der Grundausstattung und internationale Kooperationen gelang es, die Leistungsfähigkeit erheblich zu verbessern. So konnte auch in Journalen mit hohem ImpactFaktor publiziert werden. Allerdings bedarf eine solche Ent- wicklung der Schaffung einer guten Basis-Ausstattung an Personal und Forschungslaboratorien.

Ist der Qualitätssprung von allen ostdeutschen UniversitätsHautkliniken gleichermaßen vollzogen worden? Von den sieben ostdeutschen Universitäts-Hautkliniken (außer Berlin) wurden 1997 - 1999 im Durchschnitt 36 Publikationen (MEDLINE-referiert) mit einem mittleren kumulativen Impact-Faktor von 39 veröffentlicht (Quotient 0,92). Überdurchschnittliche Publikationszahlen erreichten Leipzig, Magdeburg und Jena. Kumulative Impact-Faktoren von über 39 haben Magdeburg und Jena. Die höchsten Werte für den durchschnittlich erzielten Impact-Faktor pro Publikation weisen Greifswald, Magdeburg und Jena auf. Dieses Ranking bleibt erhalten, wenn nur das Jahr 1999 betrachtet wird.

Vor einer Simplifizierung in der Auslegung der Daten sei ausdrücklich gewarnt. Es gibt engagierte Wissenschaftler an allen Kliniken. Von Bedeutung für deren Entwicklung ist aber gewiss die Wissenschaftslandschaft, in der sich die einzelne Klinik findet. Offensichtlich zeichnet sich eine zunehmende Diversifikation der Entwicklungsbedingungen und des daraus resultierenden Leistungsvermögens ab.

Die Region Jena ist Dank der Bemühungen von Land, Bund, Unternehmen und Stiftungen eine besonders florierende Wissenschaftslandschaft, wovon die Dermatologie durch vielfältige Kooperationen auch besonders profitiert.

Insgesamt zeigt sich eine positive Korrelation von Publikationszahl und kumulativem Impact-Faktor. Höhere kumulative Impact-Faktoren werden derzeit noch stärker durch eine erhöhte Anzahl von Publikationen und nicht durch eine Dominanz der High-Impact-Journale erzielt. Es ist anzunehmen, dass in Zukunft die stärkste Differenzierung durch den Zugang zu High-Impact-Journalen und nicht durch extensive Steigerung der Publikationszahlen zu erwarten ist.

\section{Literatur}

${ }^{1}$ Dubin D, Arndt KA. The impact of dermatology journals. Arch Dermatol 1995; 131: 1059-1060

2 Eisenhofer G. Scientific productivity: Waning importance for career development of todays scientist? http://www.his.com/ q graeme/pandp.htlm.

${ }^{3}$ Fröhlich G. Von der Macht des Messens. heureka 1/99 über: http://www.falter.at/heureka/archiv/99_1/03macht.htm.

${ }^{4}$ Garfield E. Journal impact factor: a brief review. Can Med Assoc J 1999; 161: 979-989

${ }^{5}$ Garfield E. Random thoughts on citationology - its theory and practise. Scientometrics 1998; 43: 69-76

${ }^{6}$ Garfield E. The diverse roles of citation indexes in scientific research. Rev Invest Clin 1998; 50: 497-504

${ }^{7}$ Hecht F, Hecht BK, Sandberg AA. The journal ,impact factor“: a misnamed, misleading, misused measure. Cancer Genet Cytogenet 1998; 104: 77-81

${ }^{8}$ Lehrl S. Zur Ungerechtigkeit, mit Biochemikern verglichen zu werden: Warum fachspezifische Normen wichtig sind. Blick durch die Wissenschaft 1991; 2: 11-12

${ }^{9}$ Scholz A. Geschichte der Dermatologie in Deutschland. Berlin: Springer Verlag, 1999

${ }^{10}$ Stern RA, Arndt KA. Top cited authors in dermatology: a citation study from 24 journals: 1982 -1996. Arch Dermatol 1999; 135: $299-302$ 
${ }^{11}$ Wollina U. Wissenschaftliche Publizistik an der UniversitätsHautklinik Jena. Dermatol Monatsschr 1993; 179: 73-78

${ }^{12}$ Wollina U. 70 Jahre Universitäts-Hautklinik Jena. Z Dermatol 1994; 180: 58-63

${ }^{13}$ Wollina U, Bonitz R. 70 Jahre Universitäts-Hautklinik Jena - Tradition und Fortschritt. Hautarzt 1995; 46: 737-740

\section{Prof. Dr. Uwe Wollina}

Klinik für Dermatologie und Allergologie Klinikum der Friedrich-Schiller-Universität Jena Erfurter Straße 35

07740 Jena

\section{BUCHBESPRECHUNG}

Krutmann, J., H. Hönigsmann, C. A. Elmets, P. R. Bergstresser (Hrsg.): Dermatological Phototherapy and Photodiagnostic Methods. 412 Seiten, $104 \mathrm{Abb}$. (davon 50 in Farbe), 71 Tab. Springer, Berlin 2001. Geb. DM 179,--. ISBN 3 540-67789-5

Die Phototherapie und die Photochemotherapie gehören heute zu den wichtigsten Säulen der modernen dermatologischen Therapie. Dies beruht insbesondere auf der vor allem seit Mitte der 70er Jahre begonnenen intensiven Zusammenarbeit von Physikern, Ingenieuren, Biologen und Dermatologen, welche zur systematischen Entwicklung neuer Formen der Photo(chemo)therapie, wie PUVA, UVB-311 nm Schmalspektrum, UVA-1 Hochdosis und der photodynamischen Therapie geführt hat. Bei dem vorliegenden Buch handelt es sich um mehr als nur die Übersetzung des „Handbuch der dermatologischen Phototherapie und Photodiagnostik“ (Herausgeber: J. Krutmann und H. Hönigsmann) ins Englische; die Herausgeber wurden um die renommierten U.S.-amerikanischen Photodermatologen Craig Elmets und Paul Bergstresser ergänzt. Ebenso wurden die meisten Kapitel gründlich überarbeitet, unter Mitarbeit weiterer international bekannter Autoren. Das übersichtliche und umfassende Grundkonzept mit Kapiteln zu wissenschaftlichen Grundlagen, Photo(chemo)therapie in der Praxis, speziellen phototherapeutischen Verfahren, Photoprotektion in der Praxis, Photodiagnostik in der Praxis sowie einem Anhang mit Richtlinien wurde dabei weitgehend beibehalten. Diese Gliederung offenbart ein klares Konzept der Herausgeber und Autoren, die sich in dem vorliegenden Werk gleichermaßen um Darstellung des wissenschaftlichen Hintergrundes der Photobiologie sowie die Vermittlung klinischer, praxisorientierter Informationen und Richtlinien bemühen. Eindrucksvoll wird die Entwicklung der „konzeptgebundenen Photomedizin" dargestellt, welche auf dem Boden wissenschaftlich definierter pathophysiologischer und photobiologischer Grundlagen zur Einführung von effektiveren Therapieschemata wie auch zu einer deutlichen Erhöhung der Patientensicherheit geführt hat. Die klinisch orientierten Kapitel sowie der exzellente Anhang bieten für jede Therapiemodalität klare Indikationen und Richtlinien zur praktischen Durchführung. Eine Vielzahl von übersichtlichen Tabellen und Abbildungen tragen wesentlich zum Verständnis der zum Teil komplexen Inhalte bei. Dieses Buch setzt aufgrund seiner grundlegenden „state-of-theart" Beiträge international anerkannter Photodermatologen Maßstäbe als Nachschlagewerk und praxisorientierter Leitfaden. Grundkenntnisse des Englischen vorausgesetzt, ist seine Lektüre ein „Muss“ für forschende Photodermatologen wie für den in der Phototherapie tätigen Kliniker. Aufgrund der ausführlich behandelten praktischen Anleitungen und Informationen zu technischer Ausstattung, Photodiagnostik und Phototherapie eignet sich das vorliegende Buch insbesondere auch für den in der Praxis tätigen Dermatologen.

J. Thiele, Jena 